\title{
Influence of the diet on the turnover of bile acids in germ-free and conventional rats
}

\author{
By B. E. GUSTAFSSON \\ Department of Germfree Research, Karolinska Institutet, Stockholm, Sweden \\ AND A. NORMAN \\ Department of Clinical Chemistry, Danderyds Sjukhus, Danderyd, Sweden
}

(Received 24 June I968-Accepted 6 December 1968)

\begin{abstract}
I. $\left[24^{-14} \mathrm{C}\right]$ cholic acid was given to conventional and germ-free rats and the faecal isotope excretion studied for 8 days. The turnover, pool size and daily excretion of cholic acid and its metabolites were calculated for rats on three different diets, i.e. a commercial type diet (pellets), a semi-synthetic diet without $\left(\mathrm{D}_{7}\right)$ and with $20 \%$ cellulose $\left(\mathrm{D}_{7}+\right.$ cellulose). The transit time of the intestinal contents was evaluated by following the excretion of an orally administered dose of carmine.

2. The amount of bile acids excreted was two to three times higher in both conventional and germ-free rats receiving pellets than in those receiving diet $\mathbf{D}_{7}$. This difference in bile acid excretion between animals receiving different diets cannot, therefore, be caused by an influence of the diet on the gastro-intestinal microflora. This effect of the commercial diet could not be due to a high fibre content, since an increase in the fibre content of the sernisynthetic diet $\mathrm{D}_{7}$ by the addition of $20 \%$ cellulose did not reproduce the effect obtained with the commercial diet.

3. The difference observed in bile acid excretion between germ-free and conventional rats on diet $\mathrm{D}_{7}$ was mainly ascribed to the much longer transit time of intestinal contents in germfree as compared with conventional rats.
\end{abstract}

The turnover of cholic acid can be calculated by determining the rate of faecal isotope excretion after administration of $\left[24^{-14} \mathrm{C}\right]$ cholic acid. Studies using this technique in the rat have demonstrated that both the intestinal flora and the diet influence the elimination of cholic acid. The slowest faecal excretion rate of labelled cholic acid has been observed in germ-free rats (Gustafsson, Bergström, Lindstedt \& Norman, I957), the estimated biological half-life of cholic acid being at least three times that in conventional rats on the same semi-synthetic diet (D 7$)$. Determination of faecal excretion products of cholic acid gave a mean daily value of $\mathrm{I} .9 \mathrm{mg}$ in germ-free rats and $5^{\circ} \mathrm{I} \mathrm{mg}$ in conventional rats (Gustafsson, Normal \& Sjövall, I960). Portman \& Murphy (1958) have demonstrated that the type of diet has a pronounced influence on the turnover of cholic acid in conventional rats. In rats fed on Purina chow, the biological half-life of cholic acid was $2 \cdot 0$ days, whereas with a standard, low-residue, semi-purified diet with sucrose as the source of carbohydrate, and with the same diet supplemented with $20 \%$ of cellulose, the half-lives were 4.2 and $I^{\circ} 4$ days, respectively. They also found that the mean faecal excretion of cholate by animals given Purina chow was nearly five times that of animals given the sucrose synthetic diet.

Factors associated both with the microflora in the gastro-intestinal tract and with the diet ingested play an important part in the metabolism of bile acids, and, in the conventional animal, these interdependent factors can affect various conditions per- 
taining to the formation and passage of bile acids through the body. For example, the rate of elimination of bile acids may be influenced by an effect on their synthesis in the liver; the reabsorption of bile acids from the intestine may be influenced by variations in the formation of their different metabolites, present in different physical states in the contents of the gastro-intestinal tract, and also by alterations in the transit time of these contents due to changes in the muscular activity of the intestinal wall, combined, in rodents, with an effect on the practice of coprophagy.

In the present investigation germ-free and conventional rats receiving the same three diets have been compared, in order to study the influence of the diet per se on the turnover of bile acids. Two of these diets were semi-synthetic, one being low and the other high in fibre; a third, commercial diet, with a comparatively high fibre content, was also used. The transit time of the gastro-intestinal contents was studied by administering carmine; coprophagy was prevented in some of the rats.

\section{EXPERIMENTAL AND RESULTS}

The following systematic names are given to compounds referred to in this report by trivial names: cholic acid, $3 \alpha, 7 \alpha$, I $2 \alpha$-trihydroxy- $5 \beta$-cholanoic acid ; chenodeoxycholic acid, $3^{\alpha}, 7^{\alpha}$-dihydroxy- $5 \beta$-cholanoic acid; deoxycholic acid, $3 \alpha, \mathrm{r} 2 \alpha$-dihydroxy$5_{5}^{\beta}$-cholanoic acid.

\section{Animals}

Germ-free rats of the Swedish strain were reared according to the technique described earlier (Gustafsson, 1948, 1959). All the rats were male and their initial ages ranged from 107 to 235 days.

\section{Diet and treatment of animals}

All the germ-free animals were housed in metabolism cages in one jacket isolator made of plastic and stainless-steel. After the experiments had been completed with the germ-free animals, the conventional rats were transferred to this isolator and housed in the same manner. Three weeks before the experiments began, the rats were put into metabolism cages, divided into three groups and fed the experimental diets. The first group received the basal semi-synthetic diet, $D_{7}$ (Table I), the second group a diet made up of 4 parts of diet $\mathrm{D}_{7}$ to I part of cellulose (Cellulose Powder for chromatography; Kebo AB, Stockholm, Sweden), and the third group, a pelleted commercial diet. According to the analysis supplied by the manufacturers, the composition of the pellets was ( $\%$ by weight): protein, 27.5 ; fat, ro.5; nitrogen-free extract, $41 \cdot 0$; ash, $9 \cdot 0$; fibre, 4.7 ; water, $7 \cdot 3$. All diets were sterilized by autoclaving for 20 min at $121^{\circ}$. Diets and water were given $a d$ lib. From the beginning of the experiments coprophagy was prevented in half of the rats in each dietary group, by restraining cages similar to those described by Bollman, Cain \& Grindlay (r948). On the ist day of the experiment, $0 . \mathrm{I}$ mg sodium $\left[24^{-14} \mathrm{C}\right.$ ]cholate $(5 \mu \mathrm{c})$, autoclaved in I ml water, was given orally to each rat by means of a stomach tube. Through the same stomach tube, a suspension of $100 \mathrm{mg}$ carmine in $\mathrm{I} \mathrm{ml}$ water was given. Faeces and urine were collected every 24 h. After 8 days the rats were killed. The liver, stomach, small intestine (divided 
into three equal segments), caecum and large intestine were removed separately. The stainless-steel funnels of the metabolism cages were renewed every day and removed from the isolator. After the faeces had been collected, the surfaces of the funnels were washed with $80 \%$ ethanol. At the end of the experiment the whole metabolism cage was washed in the same manner.

\section{Table I. Composition of the basal diet $D_{7}$ (per kg diet)}

\begin{tabular}{lr}
\multicolumn{1}{c}{ Ingredient } & Amount \\
Casein & $220 \mathrm{~g}$ \\
Wheat starch & $635 \mathrm{~g}$ \\
Arachis oil & $100 \mathrm{~g}$ \\
Salt mixture* & $40 \mathrm{~g}$ \\
Ascorbic acid & I g \\
Inositol & I g \\
Choline & $2 \mathrm{~g}$ \\
Thiamine & $50 \mathrm{mg}$ \\
Riboflavine & $20 \mathrm{mg}$ \\
Pyridoxine & $20 \mathrm{mg}$ \\
Nicotinamide & $200 \mathrm{mg}$ \\
Calcium pantothenate & $100 \mathrm{mg}$ \\
p-Aminobenzoic acid & $300 \mathrm{mg}$ \\
Biotin & I mg \\
Folic acid & $20 \mathrm{mg}$ \\
Cyanocobalamin & $0.02 \mathrm{mg}$ \\
Vitamin A (palmitate) & $21000 \mathrm{i} . \mathrm{e}$. \\
Vitamin D (ergocalciferol) & $4500 \mathrm{i} . \mathrm{u}$. \\
Vitamin K (phylloquinone) & $10 \mathrm{mg}$ \\
Vitamin E (tocopheryl acetate) & $500 \mathrm{mg}$ \\
$\quad *$ Hubbell, Mendel \& Wakeman (I 937$).$
\end{tabular}

\section{Extraction of labelled bile acids}

Faeces, liver, stomach and intestinal segments were each homogenized in 4 parts of water with a Virtis Homogenizer with propeller for I min at $5000 \mathrm{rev} / \mathrm{min}$. Ethanol was added to the homogenate to give a final concentration of $80 \%$ (v/v) ethanol. After refluxing twice for $3 \mathrm{~h}$, the residue was further extracted for $48 \mathrm{~h}$ with boiling chloroform-methanol 1 : I $(\mathrm{v} / \mathrm{v})$. Radioactivity was determined in a Packard Tri-Carb Scintillation counter. Internal standards were always used to correct for quenching.

The amount of faecal labelled bile acids that remained after extraction with $80 \%$ ethanol was calculated by determining the amount of isotope recovered in the subsequent chloroform-methanol extracts. As shown in Table $2, \mathrm{I} \cdot 7$ to $8.2 \%$ of the labelled faecal bile acids were not recovered in the $80 \%$ ethanol extract from both germ-free and conventional rats, irrespective of the diet. Furthermore, it is interesting to note that there were only small differences between germ-free and conventional rats and between the different dietary groups.

After determining the isotope remaining in the liver and the gastro-intestinal tract, and the isotope in the faeces excreted during the experimental period, the recovery of the isotope as a percentage of the amount given was calculated (Table 3 ). The mean values for the recovered isotope were consistently lower in the germ-free (86-89\%) than in the conventional rats $(90-95 \%)$. The excretion of labelled compounds in 
urine was also determined. Labelled compounds could be demonstrated in only three rats, but the total urinary excretion of isotope was never above $0.1 \%$ of the isotope given. The $80 \%$ ethanol washings of funnels and cages were refluxed and filtered. In no instance did the extracts contain labelled compounds.

Table 2. Percentage of administered isotope recovered by extraction from the liver and gastro-intestinal tract of rats 8 days after oral administration of $\left[24^{-14} \mathrm{C}\right]$ cholic acid, and from the faeces accumulated during this period

$\begin{array}{lccc}\text { Rearing conditions } & \text { Diet } & \overbrace{\text { Mean* }}^{\text {Isotope recovered }} & \text { Range } \\ \text { Germ-free } & \mathrm{D}_{7} & 89 & 88-92 \\ \text { Conventional } & \mathrm{D}_{7} & 94 & 91-98 \\ \text { Germ-free } & \mathrm{D}_{7}+\text { cellulose } & 88 & 83-90 \\ \text { Conventional } & \text { D7 +cellulose } & 95 & 92-99 \\ \text { Germ-free } & \text { Pellets } & 86 & 83-92 \\ \text { Conventional } & \text { Pellets } & 90 & 87-94 \\ * \text { Mean value for three rats with and three without prevention of coprophagy. }\end{array}$

Table 3. Amount of labelled bile acids extracted with chloroform-methanol $\mathrm{I}: \mathrm{I}$ from the residue obtained after initial extraction with $80 \%$ ethanol from faeces of rats

\begin{tabular}{|c|c|c|c|c|}
\hline \multirow[b]{2}{*}{ Rearing conditions } & \multirow[b]{2}{*}{ Diet } & \multirow{2}{*}{$\begin{array}{c}\text { No. of } \\
\text { extractions }\end{array}$} & \multicolumn{2}{|c|}{$\begin{array}{l}\text { Percentage of recovered } \\
\text { isotope present in } \\
\text { chloroform-methanol extracts }\end{array}$} \\
\hline & & & Mean & Range \\
\hline Germ-free & $\mathrm{D}_{7}$ & 32 & $3 \cdot 6$ & I. $7-4 \cdot 8$ \\
\hline Conventional & $\mathrm{D}_{7}$ & 48 & 47 & $3 \cdot 5-7 \cdot 0$ \\
\hline Germ-free & $\mathrm{D}_{7}+$ cellulose & 40 & $6 \cdot 7$ & $5 \cdot 2-7 \cdot 7$ \\
\hline Conventional & $\mathrm{D}_{7}+$ cellulose & 40 & $4 \cdot 8$ & $2 \cdot 9-8 \cdot 2$ \\
\hline Germ-free & Pellets & 40 & 47 & $3 \cdot 4-6 \cdot 2$ \\
\hline Conventional & Pellets & 48 & $2 \cdot 6$ & $1 \cdot 8-3 \cdot 1$ \\
\hline
\end{tabular}

\section{Distribution of remaining labelled bile acids in the different parts of the gastro-intestinal tract and liver}

Fig. I gives the percentage of labelled compounds in the liver, stomach, upper, middle and lower third of the small intestine, caecum and colon 8 days after administration of $\left[24^{-14} \mathrm{C}\right]$ cholic acid. More than $75 \%$ of the isotope recovered from the various organs investigated, from both conventional and germ-free rats, was present in the small intestine, irrespective of the diet. The upper third of the small intestine, including the duodenum, only contained $20 \%$ of the isotope present, whereas the remaining $80 \%$ was equally distributed between the middle and lower thirds. The amount of labelled bile acids in the liver and bile ducts varied between 0.7 and $5.9 \%$. The mean values for the six groups varied between $\mathrm{x} \cdot 3$ and $3.9 \%$. Labelled bile acids were present in the stomach of all conventional rats and of all germ-free rats, except for four animals. The highest value, $8.8 \%$, was found in a conventional rat kept in a restraining cage. The amounts present in the stomachs of rats in which coprophagy 
was prevented were about the same as those in the groups of animals not kept in restraining cages. The caecum contained between $2 \cdot \mathrm{I}$ and $20.5 \%$ of the labelled bile acids. No significant difference in the amount present in the caecum was observed between different groups of animals kept on different diets, or between germ-free and conventional animals. Only trace amounts of labelled compounds were found in the colon. Mean values for the different groups varied between 0.8 and $2.6 \%$.

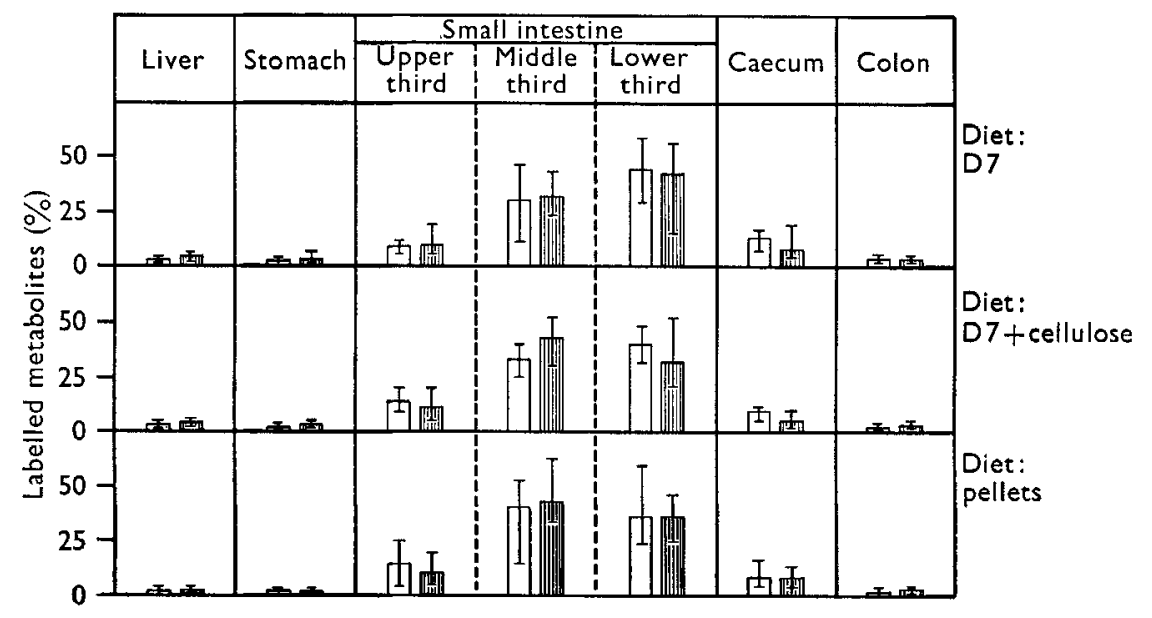

Fig. I. Percentage distribution of labelled bile acids remaining in the liver and different parts of the gastro-intestinal tract of rats 8 days after administration of $\left[24-{ }^{14} \mathrm{C}\right]$ cholic acid. Each bar gives the percentage of labelled metabolites and the vertical lines indicate range. $\square$, germ-free rats; $\mathbb{\text { ll }}$, conventional rats.

\section{Elimination rate and turnover of cholic acid}

The daily excretion of isotope was determined during 8 days following administration of $\left[24^{-14} \mathrm{C}\right]$ cholic acid. Fig. 2 gives semi-logarithmic plots of $-\log \left(\mathrm{x}-\left[u_{t} / a_{0}\right]\right)$ $v$. time, where $u_{t}=$ cumulative excretion of isotope, to time $t$; and $a_{0}=$ administered amount of isotope. Graphing resulted in straight-line plots which intersected the zero ordinate at about 0.5 day. The half-life times obtained from these graphs (Fig. 2) are given in Fig. 3 .

As the elimination of cholic acid from the pool probably follows first order kinetics, this equation will hold:

$$
a_{f}=a_{0} \mathrm{e}^{-k t},
$$

where $a_{0}=$ given amount of isotope, $a_{f}=$ isotope remaining in the pool at the time $t$, and $k=$ elimination constant. As the bile acids in the rat are present only in the liver and the gastro-intestinal tract, and only in trace amounts in the rest of the animal (Sjövall \& A kesson, 1955), it is assumed that the pool is equal to the isotope recovered when the rats were killed. From the values of $k$ the biological half-life was calculated according to

$$
t_{2}=\log 2 / k .
$$


However, the isotope given was not completely recovered. It is not known whether this was due to losses during sampling or to incomplete extraction and so it is impossible to evaluate which of the two calculated values for half-life is correct. However, the differences between the two estimations are small and do not interfere with the validity of the following conclusion.

Figs. 2 and 3 show that, in germ-free rats, the biological half-life of cholic acid, in animals reared on pellets, was only a third of that observed in animals on diet $\mathrm{D}_{7}$. Addition of cellulose to diet $\mathrm{D}_{7}$ shortened the half-life time of cholic acid, and in animals receiving pellets it was even shorter than in those on the semi-synthetic diets. In

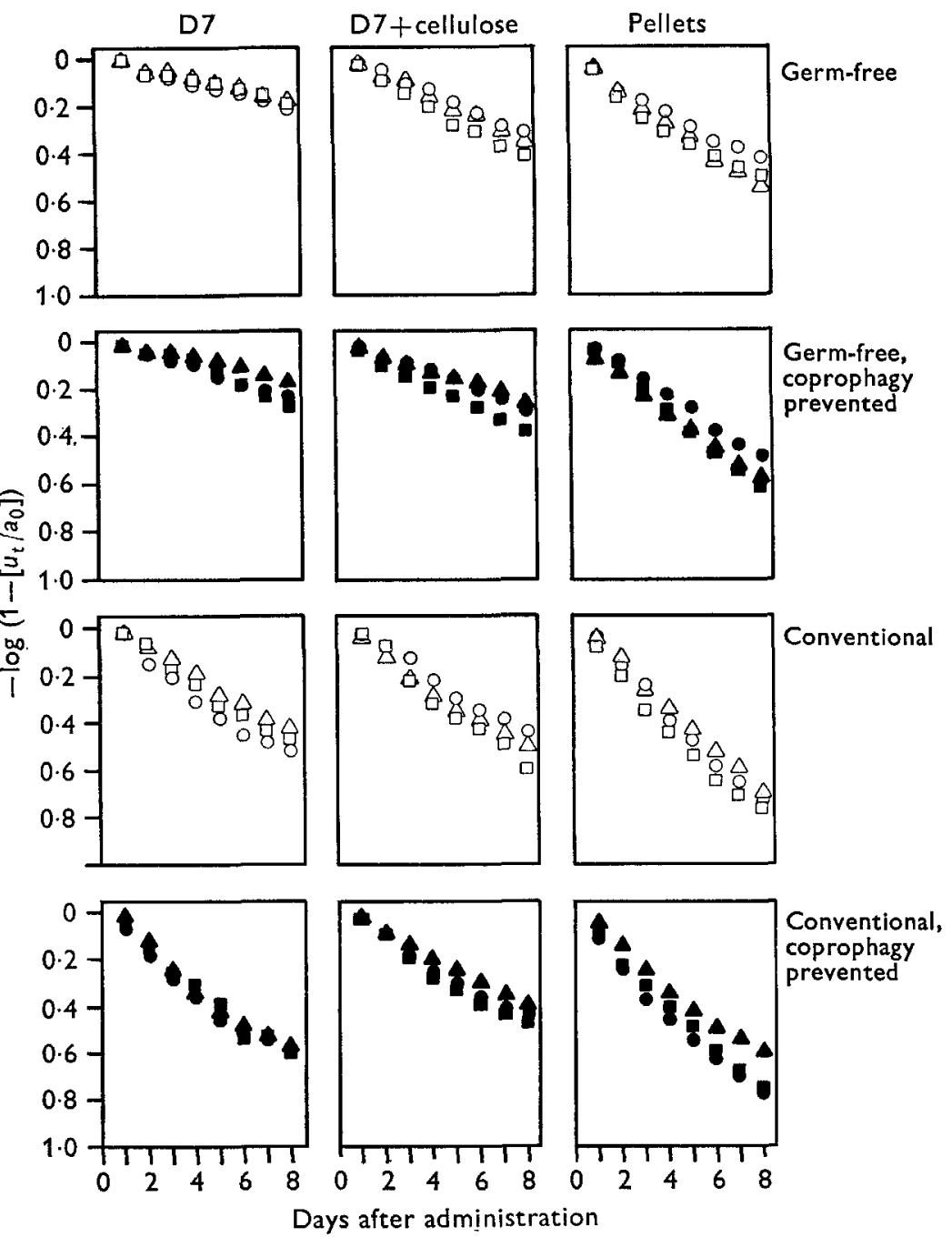

Fig. 2. Semi-logarithmic plot of elimination of $\left[2^{-14} \mathrm{C}\right]$ cholic acid in rats given diet $\mathrm{D}_{7}$, $\mathrm{D}_{7}+$ cellulose, or pellets. $u_{t}=$ cumulative excretion of isotope to time $t ; a_{0}=$ administered amount of isotope; $O, \triangle, \square$, represent individual rats reared in ordinary metabolism cages; $\boldsymbol{\bullet}, \mathbf{\Delta}, \mathbf{E}$, represent individual rats in which coprophagy was prevented by restraining cages. 
conventional rats the half-life of cholic acid in animals receiving pellets was slightly shorter than in those receiving diet $\mathrm{D}_{7}$, but the diet did not exert the same influence as that observed in germ-free rats, since the cellulose supplement to diet $D_{7}$ did not shorten the half-life of cholic acid. The elimination rates of cholic acid in germ-free rats were always slower than in conventional rats on all the diets tested. The greatest

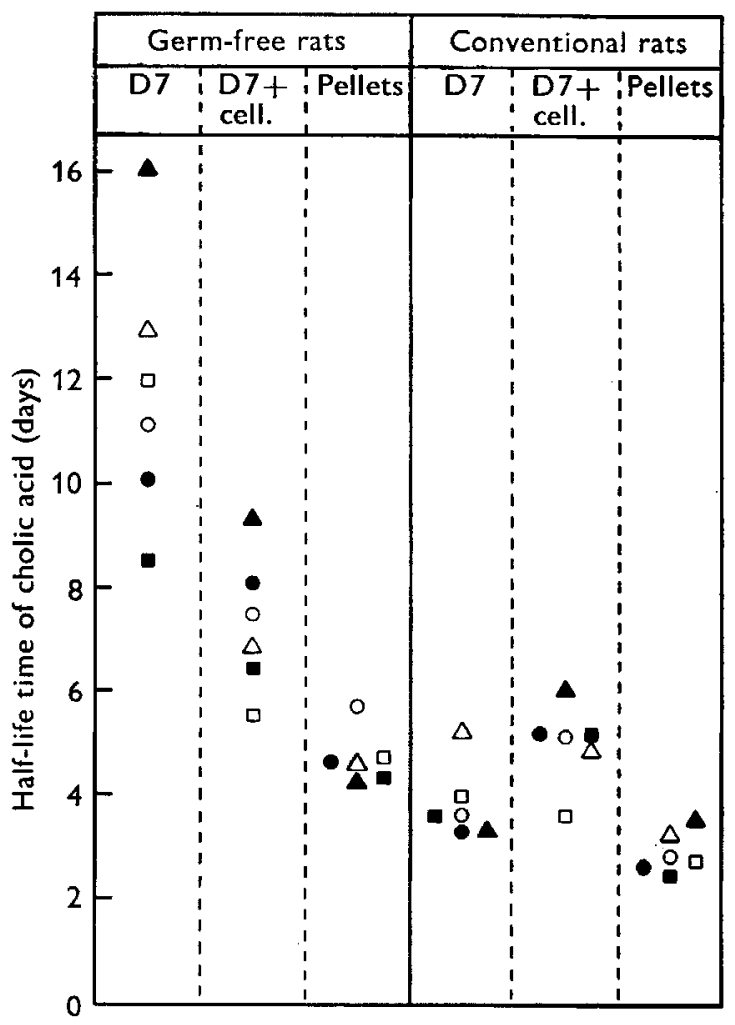

Fig. 3. Half-life times of $\left[24^{-14} \mathrm{C}\right]$ cholic acid in rats reared on diet $\mathrm{D}_{7}, \mathrm{D}_{7}+$ cellulose (D $7+$ cell.), or pellets. For symbols see Fig. 2 .

differences between germ-free and conventional rats were observed in rats reared on diet D 7 . Prevention of coprophagy had no obvious influence on the half-life of cholic acid in any of the groups.

\section{Determination of cholic acid and its metabolites present in the liver and intestinal tract}

The extracts of labelled bile acids from the small intestine were combined and hydrolysed in $\mathrm{N}-\mathrm{NaOH}$ for $6 \mathrm{~h}$ at $120^{\circ}$ in a sealed tube. The unconjugated bile acids were extracted with diethyl ether from an acidified solution and separated by column chromatography by the method described earlier (Gustafsson et al. 1960). The chromatographic fractions containing cholic acid were combined and the amount quantitated enzymically by means of a NAD-linked $\beta$-steroid dehydrogenase prepared from 
Pseudomonas testosteroni, as described by Iwata \& Yamasaki (I964). The specific radioactivity of cholic acid was determined.

Assuming that the specific radioactivity of cholic acid is equal to that of its metabolites (Gustafsson et al. 1960), and that the specific radioactivity was the same in different parts of the intestinal tract at the time the rats were killed, the total amount of cholic acid plus its metabolites can be calculated by dividing total radioactivity in the rat by the specific radioactivity of cholic acid in the small intestine.

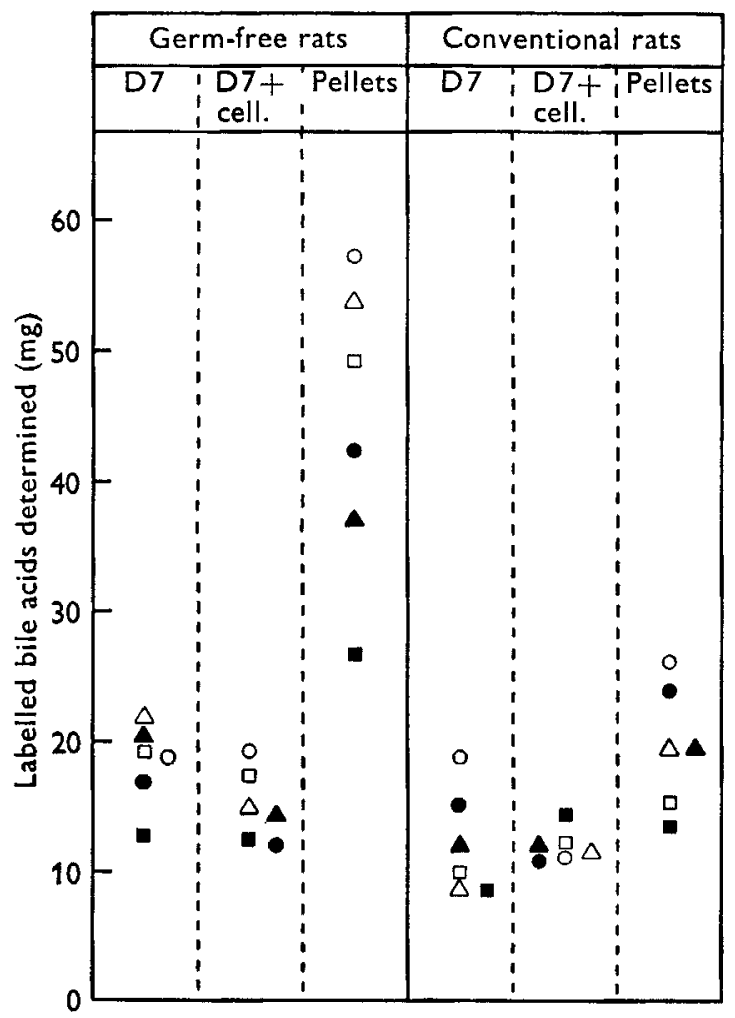

Fig. 4. Estimated amount of cholic acid and its metabolites present in liver and intestinal tract of rats receiving diet $\mathrm{D}_{7}, \mathrm{D}_{7}+$ cellulose $\left(\mathrm{D}_{7}+\right.$ cell.), or pellets. For symbols see Fig. 2.

The estimated amounts of cholic acid and its metabolites are summarized in Fig. 4. Mean values of $18.5,15^{\circ} \circ$ and $44^{\circ} 5 \mathrm{mg}$ labelled bile acids were found in the intestinal contents of germ-free rats reared on diets $\mathrm{D}_{7}, \mathrm{D}_{7}$ with cellulose, and pellets, respectively; the corresponding values for conventional rats were $10.7,12 \cdot 1$ and $19.7 \mathrm{mg}$. Germ-free and conventional rats reared on diet $D_{7}$ and $D_{7}$ with cellulose were found to contain about the same amount of labelled bile acids. However, when the animals were receiving pellets germ-free rats contained approximately double the amount of bile acids found in conventional rats. 


\section{Daily excretion of cholic acid and its metabolites}

The turnover time, $\bar{T}$, of cholic acid was calculated from the formula

$$
\bar{T}=t \frac{1}{2} / \ln 2 .
$$

$t \frac{1}{2}$ was calculated from determinations of faecal isotope excretion. The daily excretion of cholic acid and its metabolites was calculated by dividing the total amount of these compounds present in the rat by the turnover time. The values for daily excretion so determined are summarized in Fig. 5.

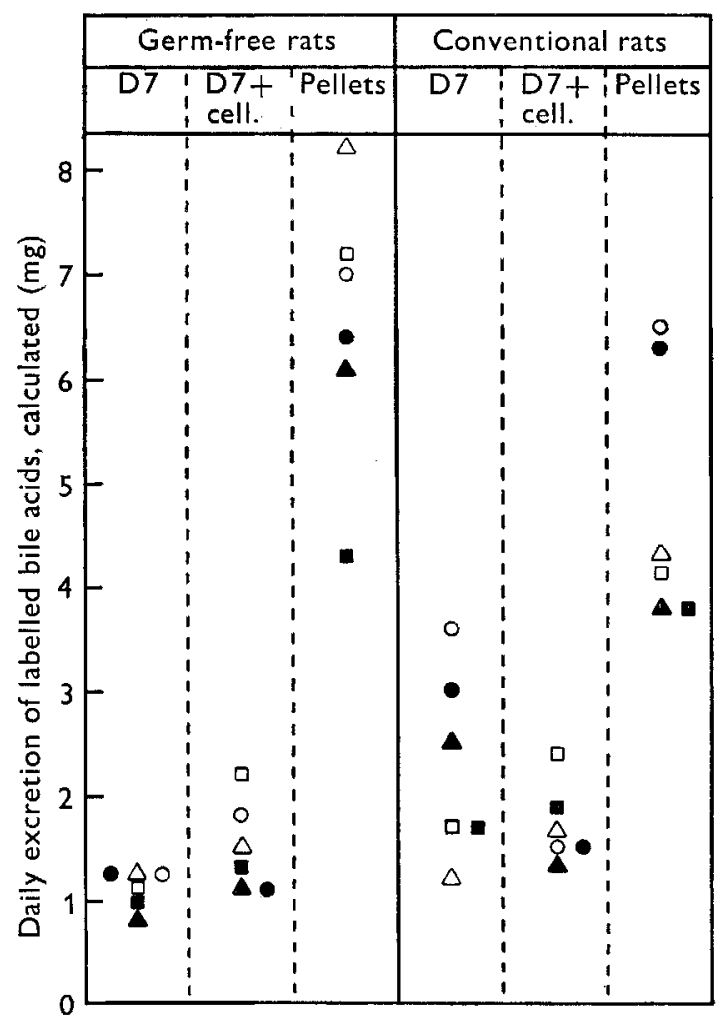

Fig. 5. Calculated values for the excretion of cholic acid and its metabolites in rats receiving diet $D_{7}, D_{7}+$ cellulose $\left(D_{7}+\right.$ cell.), or pellets. For symbols see Fig. 2 .

The average daily excretion of cholic acid and its metabolites was found to be $I \cdot I$, $\mathrm{I} \cdot 5$ and $6.6 \mathrm{mg}$ in germ-free rats reared on $\mathrm{D}_{7}, \mathrm{D}_{7}$ with cellulose, and pellets, respectively. The corresponding values for conventional rats were $2 \cdot 3, \mathrm{r} \cdot 7$ and $4 \cdot 2 \mathrm{mg}$. The addition of cellulose to $\mathrm{D}_{7}$ did not increase the bile acid excretion in either germ-free or conventional rats. Feeding with pellets resulted in a significantly higher bile acid excretion than either of the semi-synthetic diets tested.

In rats fed $D_{7}$ with cellulose, or pellets, the daily excretion was found to be the same in conventional as in germ-free rats. In animals receiving $\mathrm{D}_{7}$ the germ-free rats had a significantly lower bile acid excretion than the conventional rats. 


\section{Faecal excretion of carmine}

Carmine is widely used as a faecal marker since it is easy to detect in faecal samples. The faecal excretion of carmine was determined during the present investigation in order to study the duration of faecal excretion of an amount of food administered orally.
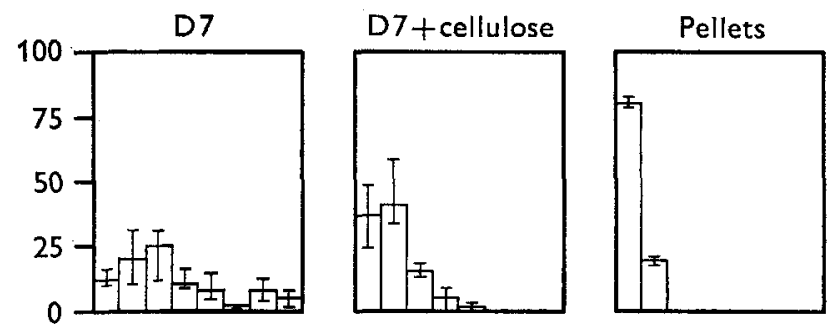

Germ-free
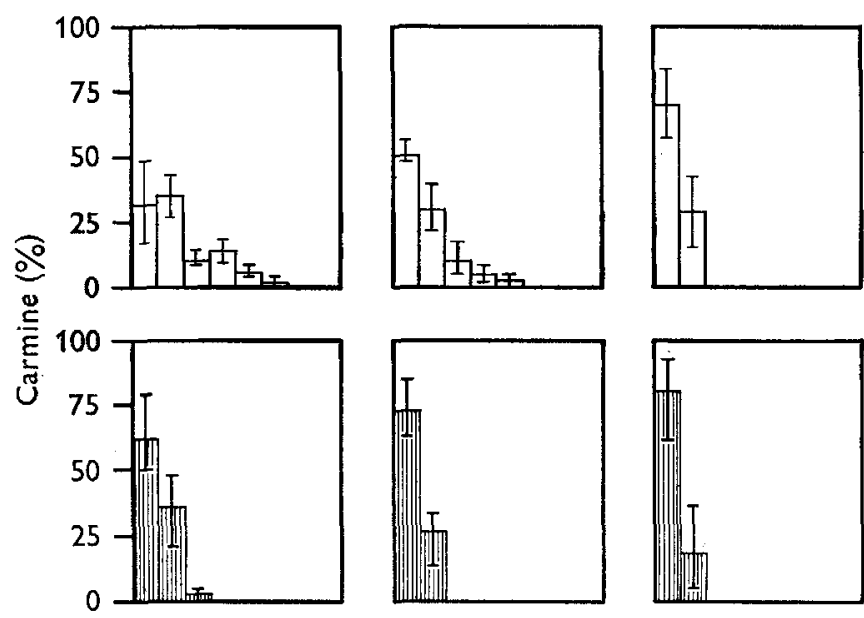

Germ-free,

coprophagy

prevented
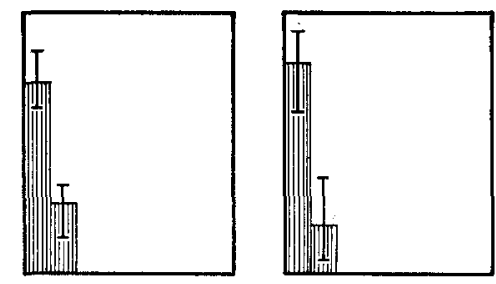

Conventional
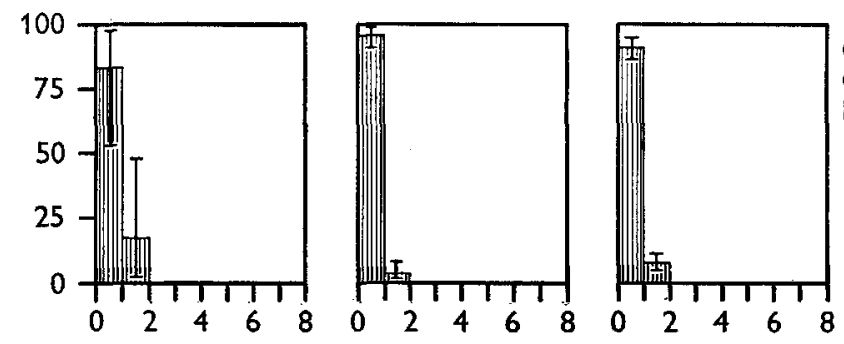

Conventional, coprophagy provented

Days after administration

Fig. 6. Carmine excreted, after oral administration, in faeces of rats given diet $D_{7}, D_{7}+$ cellulose, or pellets. Each bar gives carmine excreted daily as a percentage of the recovered amount and the vertical lines indicate the ranges.

Thefaecal residue after extraction of the labelled bile acids with chloroform methanol I: I was homogenized for $5 \mathrm{~min}$ in $\mathrm{N}-\mathrm{NaOH}$ and centrifuged at $25000 \mathrm{~g}$ for $60 \mathrm{~min}$. The absorption spectrum from 200 to $65^{\circ} \mathrm{nm}$ of the supernatant fractions was obtained by a recording spectrophotometer. The absorbance at the maximum of $525 \mathrm{~nm}$ was used to calculate the concentration of carmine. 
The daily faecal excretion of carmine is shown in Fig. 6. The conventional rats excreted more than $50 \%$ of carmine in the first $24 \mathrm{~h}$ faecal sample. Most of the remainder was excreted within $4^{8} \mathrm{~h}$. In rats reared on diet $\mathrm{D}_{7}$ a trace amount of carmine was detected in faeces $48 \mathrm{~h}$ after oral administration of carmine. The rapid excretion of carmine, and the long sampling intervals used, do not allow any conclusions to be made concerning differences in carmine excretion between conventional rats fed on different diets or the effects of prevention of coprophagy on the excretion of carmine.

In germ-free rats the rate of excretion of carmine by those receiving diet $\mathrm{D}_{7}$ was extremely slow when coprophagy was not prevented, since $3^{-13} \%$ of the recovered amount of carmine was present in the intestinal contents of the small intestine, caecum and colon, even after 8 days. However, when coprophagy was prevented, all the carmine administered had been excreted in the faeces by the 7 th day. Addition of cellulose to diet $\mathrm{D}_{7}$ only slightly increased the excretion rate of carmine. However, in rats receiving this diet, no differences were observed in excretion rate of carmine between animals in ordinary metabolism cages and animals in which coprophagy was prevented. Carmine was excreted more slowly by germ-free rats than by conventional rats in the groups receiving diet $\mathrm{D}_{7}$ or $\mathrm{D}_{7}$ with cellulose. In germ-free and conventional rats receiving pellets, the same, relatively fast, excretion of carmine was observed.

\section{DISCUSSION}

\section{Experimental technique}

Several different solvent systems have been described for the extraction of labelled faecal bile acids derived from $\left[24^{-14} \mathrm{C}\right]$ cholic acid. Eneroth, Hellström \& Sjövall (1968) have proved that extraction of human faeces with chloroform-methanol will give complete recovery of bile acids. In our previous investigations in the rat faeces have always been extracted with $80 \%$ ethanol as a standard procedure. It has been shown, however, in the present investigation that, although most of the labelled metabolites of cholic acid were extracted with this solvent, a further $2 \cdot 6-6 \cdot 7 \%$ could be recovered in the subsequent chloroform-methanol extract. It has been suggested (Eneroth et al. 1968) that chloroform-methanol is needed for the complete extraction of bile acids, owing to the possibility that they may be bound to micro-organisms. However, this cannot be the only explanation, since chloroform-methanol had also to be used for the complete extraction of faecal bile acids from the germ-free rats.

The percentage recovery of isotope after oral administration of $\left[24^{-14} \mathrm{C}\right]$-labelled bile acid has been studied in various animal species, and sometimes a low recovery of isotope has been reported (cf. Danielsson, I963). A recovery of approximately $90 \%$ of the isotope was obtained in the present investigation, after oral administration of $\left[24^{-14} \mathrm{C}\right]$ cholic acid, and the mean percentage was slightly higher in the conventional than in the germ-free rats. Those investigators, who obtained a low recovery of isotope after oral administration of $\left[24^{-14} \mathrm{C}\right]$-labelled bile acids, have suggested that $\beta$ oxidation of bile acids might be an explanation for loss of isotope during their experimental studies. Micro-organisms have been isolated from soil that have brought about $\beta$-oxidation of the side-chain to form bisnorcholanoic acids, i.e. bile acids without the 
carbon atoms $\mathrm{C}-23$ and $\mathrm{C}-24$, after growth in a medium with bile salt as the only carbon source (Severina, 'Torgov, Skrjabin, Wulfson, Zaretskii \& Papernaja, I968). However, in the present investigation there was a greater percentage recovery of isotope from conventional than from germ-free rats, which makes it very unlikely that $\beta$-oxidation due to microbial activity could be an explanation for the loss of isotope observed.

In rats interest has been focused on the turnover of cholic acid, as this is the main bile acid formed from cholesterol (Danielsson, I963). The quantitative faecal excretion of cholic acid and its metabolites was calculated from determinations of cholic acid and its metabolites remaining in the rat at the end of the experimental period, and the half-life of cholic acid. Owing to the fact that the chemical structures of many faecal metabolites of cholic acid are not known (Gustafsson \& Norman, 1969), a direct determination of cholic acid and its metabolites in faeces was not attempted.

\section{Qualitative aspects of bile acid metabolism}

In parallel investigations on the same animals described in the present work the physical state of bile acids in the intestinal contents of germ-free and conventional rats (Gustafsson \& Norman, 1968) and the excretion of cholic acid metabolites in conventional rats (Gustafsson \& Norman, I969) have been studied. It was shown that approximately the same metabolites of cholic acid were excreted in conventional rats whether reared on pellets or on diet $\mathrm{D}_{7}$ with cellulose. The transformation of the deoxycholate formed was significantly greater in conventional rats on these diets than in those on diet $\mathrm{D}_{7}$ alone. Also, dialysis of faecal homogenates of conventional rats showed that part of the labelled metabolites of cholic acid were excreted in a nondialysable form. 'There was a higher excretion of non-dialysable bile acids in rats on pellets than in those on $\mathrm{D}_{7}$ with cellulose or $\mathrm{D}_{7}$ alone. There was a much greater proportion of bile acids in the sediment left after centrifugation of the caecal contents with both the germ-free and conventional rats receiving pellets than with those receiving diet $\mathrm{D}_{7}$. This indicates that a larger fraction of bile acids is adsorbed to intestinal residue in rats fed on pellets than on those fed on diet $D_{7}$.

\section{Infuence of diet on bile acid excretion in germ-free rats}

The excretion of bile acids by germ-free rats fed on pellets was found to be markedly greater than that of rats on diet $D_{7}$. The transit time of intestinal contents, judged from the excretion of carmine, was markedly less in the former rats than in the latter. It is not known which components of pellets cause an increase in bile acid excretion. If the primary cause of this increase were a less efficient absorption of bile acids, and not an increase in their synthesis in the liver, it is possible that either, or both the decrease in transit time of intestinal contents and adsorption of bile acids to intestinal residues may be contributory factors. Increasing the amount of fibre in the intestinal contents by adding cellulose to the semi-synthetic diet $\mathrm{D}_{7}$ did not increase either bile acid excretion, or the transit time, to the same level as that observed in rats fed on pellets. Both the transit time and the amount of bile acids adsorbed to intestinal residue (Gustafsson \& Norman I968) were only slightly different in rats receiving pellets from the values observed in rats on diet $\mathrm{D}_{7}$ alone. 


\section{Coprophagy}

Previous studies (Gustafsson et al. 1957) showed that bile acids were excreted more slowly by germ-free than by conventional rats. One reason for this could be that the germ-free rats practise coprophagy more than the conventional ones. This possibility was, therefore, investigated in the present work by including a group of animals in which coprophagy was prevented. The results show that animals receiving diet $\mathrm{D}_{7}$ practised coprophagy to a rather large extent, since carmine was excreted for more than 8 days when coprophagy was not prevented, and only for 6 days when it was prevented. Carmine excretion was rapid in all groups of conventional rats, irrespective of the diet and was complete in $48 \mathrm{~h}$. During this investigation the interval between samples was not short enough to allow any conclusions to be drawn concerning the possible differences between the various groups of animals within the $24 \mathrm{~h}$ periods chosen.

The restraining cages used in the present investigation allowed an almost complete sampling of the faeces from rats in which coprophagy was prevented.

\section{Influence of diet on bile acid excretion in conventional rats}

In conventional rats as well as in germ-free rats there was a higher faecal excretion of bile acids in animals receiving pellets than in those receiving diet $D_{7}$. The addition of cellulose to $\mathrm{D} 7 \mathrm{had}$ only a slight effect on bile acid excretion. Since the composition of the faecal labelled metabolites of cholic acid excreted in conventional rats on both diet $\mathrm{D}_{7}$ with cellulose and on pellets was the same (Gustafsson \& Norman, 1969). it is unlikely that the high excretion of bile acids observed in rats on pellets was due to the formation of less absorbable derivatives of bile acids. It is, therefore, reasonable to assume that the greater excretion of bile acids in conventional rats compared with germ-free rats was caused by differences demonstrated in the transit time of intestinal contents, and the physical state of the bile acids in the intestinal contents, rather than by the chemical nature of the excreted bile acids.

\section{Comparison of the amount of bile acids excreted in the faeces from germ-free and conventional rats on the same diet}

In previous work (Gustafsson et al. I960) it has been shown that a greater calculated amount of bile acids was eliminated daily by conventional rats than by germ-free rats receiving diet $\mathrm{D}_{7}$. This has been confirmed in the present investigation, but in addition it has been shown that this difference between germ-free and conventional rats did not exist if $20 \%$ cellulose was added to diet $\mathrm{D}_{7}$, or if the animals were given pellets. It has also been shown earlier (Gustafsson \& Norman, I962) that most of the bile acids present in the caecal contents and faeces of conventional rats on diet $\mathrm{D}_{7}$ were present in the sediment after centrifugation. The amount of bile acids found in the sediment after centrifugation of the caecal contents was greater in conventional rats than in germ-free rats, in all the dietary groups (Gustafsson \& Norman, I968). Thus the same difference in the physical state of intestinal bile acids existed between conventional and germ-free rats, irrespective of the diet used, and so it is more likely 
that the difference in the faecal excretion of cholic acid and its metabolites between germ-free and conventional rats, fed on diet $\mathrm{D}_{7}$, was due to the longer transit time of intestinal contents in germ-free rats, rather than to differences between their physical state in the intestine of the germ-free as compared to the conventional.

\section{Pool size and turnover of $\left[24^{-14} C\right]$ cholic acid}

In previous work we have shown that a reliable estimation of the daily excretion of cholic acid and its metabolites can be performed by determining both the half-life of $\left[24^{-14} \mathrm{C}\right]$ cholic acid and the total amount of cholic acid and its metabolites in the liver and gastro-intestinal tract (Gustafsson et al. I960). The total amount of bile acids in the rats was found to vary with the diet used. In germ-free rats more than double the amount of cholic acid was present in those receiving pellets than in those fed on diet D 7. The same dietary effect was observed in conventional rats, but it was less marked. Owing to the effect of diet on the pool size of bile acids, it is necessary to emphasize that a determination of the biological half-life of cholic acid alone is not sufficient for the study of the effect of diet on the turnover of bile acids.

In the present work it was shown that, irrespective of the diet used, the bile acids in both germ-free and conventional rats were mainly present in the small intestine. Although the large caecum typical of the germ-free rodent was present in the germ-free rats on all three diets, the amount of bile acids in the caecum of germ-free rats was not significantly greater than in that of conventional rats.

This work is part of investigations supported by the Swedish Medical Research Council grants 205,206 and 602 .

\section{REFERENCES}

Bollman, J. L., Cain, J. C. \& Grindlay, J. H. (1948). F. Lab. clin. Med. 33, 1349.

Danielsson, H. (1963). In Advances in Lipid Research. Vol. 1, p. 334. [R. Paoletti and D. Kritchevsky, editors.] New York, Academic Press Inc.

Eneroth, P., Hellström, K. \& Sjövall, J. (1968). Acta chem. scand. 22, I729.

Gustafsson, B. E. (1948). Acta pathol. microbiol. scand. Suppl. 73.

Gustafsson, B. E. (1959). Ann. N.Y. Acad. Sci. 78, I7.

Gustafsson, B. E., Bergström, S., Lindstedt, S. \& Norman, A. (1 957). Proc. Soc. exp. Biol. Med. $94,467$.

Gustafsson, B. E. \& Norman, A. (1962). Proc. Soc. exp. Biol. Med. 1ro, 387.

Gustafsson, B. E. \& Norman, A. (r968). Scand. F. Gastroenterol. 3, 625.

Gustafsson, B. E. \& Norman, A. (1969). Br. F. Nutr. 23, 627.

Gustafsson, B. E., Norman, A. \& Sjövall, J. (1960). Archs Biochem. Biophys. 91, 93.

Hubbell, R. B., Mendel, L. B. \& Wakeman, H. J. (1937). F. Nutr. 14, 273.

Iwata, T. \& Yamasaki, K. (I964). \%. Biochem., Tokyo 56, 424-

Portman, O. W. \& Murphy, P. (1958). Archs Biochem. Biophys. 76, 367.

Severina, L. O., Torgov, I. V., Skrjabin, G. K., Wulfson, N. S., Zaretskii, V. I. \& Papernaja, I. B. (1968). Tetrahedron 24, 2145 .

Sjövall, J. \& Åkesson, I. (1955). Acta physiol. scand. 34, х. 